\title{
Les réactions syndicales à la libéralisation du rail entre 1991 et 1996 : une comparaison franco- allemande
}

Union reactions to railway deregulation from 1991 to 1996: The French and the German case compared

\section{Nadia Hilal}

\section{OpenEdition \\ Journals}

Édition électronique

URL : https://journals.openedition.org/rhcf/173

DOI : $10.4000 /$ rhcf. 173

\section{Éditeur}

Rails \& histoire

\section{Édition imprimée}

Date de publication : 1 décembre 2007

Pagination : 368-385

ISSN : 0996-9403

Référence électronique

Nadia Hilal, « Les réactions syndicales à la libéralisation du rail entre 1991 et 1996 : une comparaison franco-allemande », Revue d'histoire des chemins de fer [En ligne], 36-37 | 2007, mis en ligne le 10 mai 2011, consulté le 22 avril 2022. URL : http://journals.openedition.org/rhcf/173 ; DOI : https://doi.org/ $10.4000 /$ rhcf.173 


\title{
Les réactions syndicales à la libéralisation du rail entre 1991 et 1996 : une comparaison franco-allemande
}

\author{
Nadia Hilal \\ ATER en science politique à l'université de Rouen
}

Au début de la décennie 1990, le transport ferroviaire reste encore largement hors du champ d'influence des politiques communautaires. Il a fallu attendre 1985 et un arrêt de la Cour européenne de justice pour envisager la préparation du rail à l'échéance du marché unique de 1993. La directive 440 de $1991^{1}$ entendait redéfinir entièrement les rapports qu'entretenaient les États avec leurs compagnies. Progressivement, la déréglementation modifiait le paysage des salariés en Europe. La naissance d'une concurrence à l'échelle européenne bouleversait les représentations des syndicats. Depuis plus d'un siècle, le cadre national semblait un horizon indépassable et le monopole public la règle dans tous les États depuis $1945^{2}$. Surtout, ces libéralisations économiques impliquaient des restructurations profondes.

Pour la première fois dans leur histoire, tous les salariés du rail en Europe étaient liés par un texte commun, la directive 91/440. Ce début d'unification de politiques nationales pouvait théoriquement constituer un point de départ dans le rapprochement syndical et la mise en place de coopérations transnationales entre organisations. Avec l'adoption de mesures de déréglementation, un avenir commun des salariés se profilait et il apparaissait désormais lié aux décisions des instances communautaires. Ce premier transfert de compétences au niveau européen a-t-il engendré un changement dans les stratégies européennes des syndicats? Autrement dit, peut-on identifier des points de convergence dans la manière dont ils ont appréhendé cette nouvelle donne? Il s'agit d'examiner si des appréciations communes sur les premiers textes communautaires ont pu contribuer à amorcer une solidarité transnationale.

1- La directive 91/440/CEE du Conseil sur le développement des chemins de fer communautaires du 29 juillet 1991.

2- Les compagnies ont toutes été nationalisées et des sociétés nationales sont créées : en 1905 en Italie, en 1926 pour la SNCB, en 1937 pour la SNCF, en 1948 pour British Rail. En Allemagne, une compagnie d'État est crée dès la seconde moitié du XIX siècle. 
Cela revient à se demander si une contrainte communautaire commune suffit à étendre aux dimensions de l'Union la fonction traditionnelle du syndicalisme, qui est d'unifier et d'homogénéiser les intérêts et représentations de différents groupes sociaux et nationaux.

Une analyse des réactions syndicales, de 1991 à 1996, nous montre des lectures radicalement opposées des enjeux communautaires. Les divergences culturelles et idéologiques ont conduit à des appréciations et à des stratégies souvent inconciliables. L'importante hétérogénéité syndicale en Europe, conjuguée à la forte diversité qui caractérise la Communauté en matière de transport (les disparités nationales dans les moyens de transports, dans les besoins, dans l'utilisation des différents modes, dans la couverture du territoire, etc.), a conduit à des réactions syndicales incompatibles. Des référentiels culturels et des conceptions politiques divergentes sur ce que doit être une politique des transports se sont ajoutés aux divergences structurelles initiales en termes d'intérêts nationaux.

La directive 91/440 constitue la première grande étape de la réorganisation du rail en Europe. Une des raisons principales invoquées pour justifier cette réorientation politique est le déclin constant de ses parts de marché, dans tous les pays européens. En 1970, le rail représentait $31,7 \%$ des marchandises transportées (contre $48,6 \%$ pour la route) ; en 1990 , ce chiffre est tombé à 18,9\% (contre 67,5\% pour la route) ${ }^{3}$. Presque les deux tiers du fret en Europe sont transportés par camion. Dans un contexte de développement des échanges, les entreprises souhaitaient bénéficier d'une offre de transport à prix modéré pour accroitre leurs exportations. La question d'une baisse du prix du transport devient d'autant plus cruciale qu'approche l'échéance du marché unique de 1993. Le transport ferroviaire, pertinent pour des distances supérieures à $500 \mathrm{~km}$, se trouve investi d'une nouvelle mission : accroittre la libre circulation des marchandises en Europe. Cette nouvelle exigence frappait les réseaux de chemins de fer de vétusté. Ceux-ci ont été pensés et construits dans un cadre national. Par conséquent, les trains circulent difficilement d'un pays à l'autre : il y a en Europe trois écartements de voie dissemblables, cinq tensions différentes, ainsi que douze systèmes de signalisation difficilement compatibles. Les trains sont arrêtés aux frontières où les locomotives et le personnel sont échangés, ce qui implique des pertes de temps considérables. Un administrateur de la Commission souligne qu'il faut plus d'un mois pour obtenir un simple prix de transport de fret ferroviaire entre deux pays européens ${ }^{4}$.

3- Marie-Madeleine Damien, La Politique européenne des transports, Paris, PUF, 1999, p. 3.

4- Entretien à Bruxelles, en 2002. 
La directive 91/440/CEE instaure l'autonomie de gestion des compagnies. Celles-ci doivent être gérées selon les mêmes principes que les sociétés commerciales afin d’améliorer leur productivité. Cette évolution implique que l'État compense les charges de service public. Les compagnies devront désormais opérer de manière « indépendante » des pouvoirs nationaux. La directive énonce le principe de l'assainissement financier des compagnies. La directive impose également une séparation comptable entre la gestion de l'infrastructure et l'activité de transporteur ('exploitation). L'infrastructure reste un monopole naturel tandis que la concurrence doit devenir possible pour l'exploitation. Le principe de l'accès des tiers au réseau constitue une révolution dans la gestion des compagnies. À terme, l'objectif est de créer un marché ferroviaire européen où s'acquièrent des sillons. Ce texte représente un tournant historique dans la gestion des compagnies au $\mathrm{XX}^{\mathrm{e}}$ siècle. Il heurte de plein fouet les traditions des syndicats de cheminots en Europe.

\section{Les divergences syndicales}

Face au projet européen de déréglementation, deux stratégies ont opposé les syndicats. D’un côté, les organisations françaises, belges, luxembourgeoises, italiennes, portugaises ou grecques ont dénoncé le consensus européen sur la manière de faire renaitre le trafic de fret ferroviaire. Elles ont insisté sur les effets négatifs de la future libéralisation : ses conséquences sociales bien sûr, mais aussi ses implications en matière d'investissements publics sur le long terme, d'aménagement du territoire, de survie des liaisons non rentables, de service public et enfin en matière de sécurité. Pour ce premier groupe syndical, l'introduction d'une concurrence intramodale qui s'ajoute à la concurrence entre les modes de transport ne constituait pas une solution satisfaisante à la crise du rail.

D’un autre côté, les organisations allemandes, néerlandaises, danoises ou espagnoles, après avoir manifesté de fortes réserves quant au projet de déréglementation, se sont résolues à considérer la réforme proposée comme inéluctable. Elles ont alors donné leur assentiment à la refonte du rail en négociant le passage à la libéralisation au mieux des intérêts des salariés. Ces deux stratégies opposées ont rendu impossibles pendant plusieurs années les rapprochements des points de vue syndicaux sur la politique communautaire.

Ces réponses différentes sont liées aux lignes de partage syndicales traditionnelles. En effet, il est possible de rapporter les différences d'appréciation des mesures communautaires aux divergences dans les systèmes de relations professionnelles en Europe. Face à un nouveau 
paradigme politique, le choix de la libéralisation, les syndicats du Nord disposaient de points d'appui relativement nombreux dans le système institutionnel pour faire valoir leurs intérêts. Après avoir exprimé leurs réticences, ils ont influencé les conditions d'application de la déréglementation. Les gouvernements et les partis politiques de ces pays (Allemagne, Pays-Bas, Suède, Danemark) se sont montrés soucieux de trouver le consensus politique et social le plus large possible et d'impliquer les syndicats dans le processus de réforme.

Dans le sud de l'Europe, conformément à la culture conflictuelle qui caractérise les relations professionnelles, la réforme n’a donné lieu à aucune véritable concertation sociale ou débat public approfondi. Les syndicats ont affiché leur franche opposition à la nouvelle orientation libérale des politiques ferroviaires, qualifiée d'idéologique et d'irréaliste. La résistance aux réformes communautaires était un mot d'ordre présent lors de nombreuses grèves. Les syndicats ont rejoint les compagnies dans leur stratégie d'endiguement de la réforme. La Grande-Bretagne constitue un cas particulier : l'introduction de la concurrence a été mise en œuvre intégralement et sans délai. Le Royaume-Uni est, en 1993, le seul pays en Europe à privatiser son réseau. Les syndicats ont été peu consultés ou associés aux réformes.

Dans cet article, nous étudierons les réactions des syndicats de cheminots en Allemagne et en France. Ces deux pays illustrent bien les deux stratégies syndicales antagonistes en Europe face à la directive communautaire ${ }^{5}$.

\section{La réponse syndicale d'une Allemagne réunifiée}

Les syndicats allemands ont été conduits à partager le constat de la nécessité d'une réforme. Il y a à cela des raisons culturelles et organisationnelles : une culture du consensus, l'habitude de la négociation avec la direction, un appareil syndical bien structuré, l'absence de divisions syndicales et des relations étroites avec le parti social-démocrate. À cela s'ajoutent des motifs conjoncturels : un bouleversement du paysage ferroviaire lié à la réunification.

Outre-Rhin, le projet de libéralisation est antérieur à la directive européenne de 1991. L'endettement des chemins de fer était jugé préoccupant. En 1989, une commission chargée de formuler des propositions sur l'avenir du rail constituée. Elle a permis la consultation

5- Pour une analyse détaillée des stratégies syndicales dans les autres pays européens, voir N. Hilal, «La naissance d'une coordination syndicale européenne : les mobilisations sociales dans le secteur des transports ferroviaires et routiers ", thèse, IEP de Paris, 2005 . 
de tous les acteurs intéressés au débat : les partis politiques, les syndicats, la direction de la Deutsche Bahn (DB), les Länder, les clients du rail, les usagers et les associations écologistes. Cette commission avait pour objectif d'accroitre la légitimité du projet de réforme. Elle a fait connaitre ses conclusions en décembre 1991, ce qui coïncidait avec la directive européenne sur la politique ferroviaire ${ }^{6}$. La commission allemande préconisait une nouvelle organisation ferroviaire relevant du droit privé, y compris pour le statut du personnel. La nouvelle compagnie serait délestée des pertes financières passées et du coût lié aux obligations de service public.

Dès 1989, les trois syndicats allemands de cheminots ont unanimement exprimé leur opposition de principe à la mise en place d'une concurrence intramodale. Ils ont insisté sur les dangers d'une telle ouverture, en rejetant tout projet d'une privatisation du rail. Ils ont principalement mis en avant leurs craintes d'un morcellement du rail en petites unités. Les orientations de la commission nationale étaient jugées trop libérales. Ce n'est qu'à partir de l'automne 1991, lors des tous derniers travaux, que le Gewerkschaft der Eisenbahner Deutschlands (GdED), le principal syndicat de cheminots, a fait savoir qu'il réviserait sa position si une contrepartie sociale était consentie aux salariés.

Le ralliement de ce syndicat aux vues gouvernementales a d'abord été rendu possible pour des raisons propres au système politique allemand, résumées par l'expression de "fédéralisme coopératif »" Ainsi, pour permettre la réorganisation du mode ferroviaire, un amendement constitutionnel était nécessaire, car l'article 87 de la loi fondamentale définit les fonctions de la Deutsche Bahn. Ce changement constitutionnel impliquait la construction d'un large consensus national : les deux tiers des voix au Bundestag (chambre des députés) et au Bundesrat (conseil fédéral) devaient être réunies et par conséquent le soutien de l'opposition sociale-démocrate assuré. La réforme a donc fait l'objet de longs débats.

Le Sozialdemokratische Partei Deutschlands (SPD) était attentif à l'élimination des distorsions de concurrence entre les modes de transport. Il s'est opposé à la privatisation de l'infrastructure ferroviaire. Son accord étant indispensable à la réforme, ses liens avec les syndicats ont garanti que les arguments syndicaux seraient entendus. Le SPD a montré qu'il partageait l'analyse des syndicats en mettant en avant le

6- Le gouvernement allemand l'avait accueillie favorablement.

7- Constance Grewe-Leymarie, Le Fédéralisme coopératif en RFA, Paris, Economica, 1981 ( $1^{\text {te }}$ éd.). 
sous-investissement dont pâtit le rail, la concurrence déloyale que lui livre le transport routier, en soutenant une politique ambitieuse de développement des infrastructures ferroviaires et en exigeant un certain nombre de garanties sociales.

Dans ce contexte, le GdED, syndicat majoritaire, a considéré qu'il défendrait mieux les intérêts des salariés en participant à la négociation et en formulant ses propres conditions. Par ailleurs, il aurait été difficile de s'opposer à un consensus de la société allemande sur la nécessité de réformer la DB. La stratégie du GdED a été influencée par les débats dans les autres secteurs publics et les grèves contre la réorganisation de la Deutsche Bundespost. Ces grèves, pourtant longues et suivies, ne sont pas parvenues à empêcher le changement de statut de la poste allemande. De même, la pression des milieux économiques pour demander une diminution des coûts du transport a été décisive en Allemagne. Les grandes entreprises et les ports ont dénoncé des coûts unitaires de production dans le rail parmi les plus élevés d'Europe ${ }^{8}$. Le rail devait en outre pouvoir prendre le relais d'un réseau routier national menacé de saturation. Le GdED a affirmé qu'il ne pouvait s'opposer à des conditions économiques impérieuses.

La directive communautaire a été utilisée par les partisans de la réforme pour convaincre les syndicats du fait que la déréglementation allait devenir incontournable dans les autres pays de l'Union. La directive n'était pas à l'origine d'une réforme qui avait été voulue, élaborée et discutée au niveau national bien avant, et dont les mesures dépassaient les dispositions communautaires de l'époque - comme la privatisation de la compagnie. Mais les textes européens ont contribué au ralliement des organisations à la réforme libérale du rail.

Parmi les raisons principales qui expliquent l'adhésion des syndicats à la réforme, on ne peut omettre le contexte de la réunification qui a conforté les thèses favorables à la déréglementation et offert une contrepartie symbolique et matérielle aux salariés du rail. Cet événement a constitué une opportunité unique et sans égale en Europe pour faire accepter les réformes. La réunification a constitué une "fenêtre d'opportunité $»^{9}$ qui a permis de présenter la libéralisation comme un

8- Michael Teutsch, "Regulatory reforms in the German transport sector: how to overcome multiple veto point ", in Adrienne Héritier (dir.), Differential Europe. New Opportunities and Constraints for National Policy-Making., Lanham, Rowman and Littlefield, 2001, p. 133-171.

9- John W. Kingdon, Agendas, Alternatives, and Public Policies, Boston, Little, Brown and Co., 1984. 
défi positif. Une perspective nouvelle s'offrait au rail : il s'agissait de réussir l'intégration du réseau de l'Est à celui de l'Ouest, deux systèmes séparés depuis 1945. La renaissance du rail devenait plausible : avec la fin du Bloc soviétique et le développement des échanges avec les pays de l'Est, l'Allemagne retrouvait son rang de premier pays de transit en Europe. La réunification augmentait également de $40 \%$ la superficie du territoire couvert par le rail, moyen de transport pertinent sur les longues distances. En récupérant une situation centrale en Europe, l'Allemagne devait revoir toute la structuration des transports de la RFA qui auparavant privilégiait l'axe nord-sud. Les discours gouvernementaux ${ }^{10}$ ont insisté sur cette opportunité unique à saisir, génératrice de potentiels de croissance importants qui compenseraient, à terme, les sacrifices sociaux consentis. La perspective de renaissance du rail allemand a été étayée par la grande générosité de l'État fédéral en matière d'investissements. Un plan d'investissements sur le long terme fut élaboré pour permettre au rail de regagner des parts de marché face aux autres modes de transports. L'Etat a donné de nombreuses garanties quant au financement des infrastructures et des services régionaux. Il a repris la totalité de l'ancienne dette de la DB et les obligations liées au financement des retraites. Il a fourni une aide financière importante pour moderniser les chemins de fer de l'ex-RDA et remédier à leurs nombreux maux - une administration gigantesque et bureaucratique, des effectifs pléthoriques et une faible productivité. Un fonds spécial a été constitué pour faire face au développement du chômage parmi les cheminots de l'ex-RDA et à l'existence de grandes disparités entre les anciens et nouveaux Länder. La réforme a donc pu être présentée, de façon crédible, comme la fin du fardeau financier de la DB sans que le gouvernement soit suspecté de vouloir abandonner le transport ferroviaire.

Dans cette conjoncture particulière, les défauts caricaturaux du rail est-allemand apportaient un élan supplémentaire au projet de réforme. Ils offraient une crédibilité aux discours stigmatisant le monopole ferroviaire. Ils donnaient à la réforme un sens nouveau : le rail se devait d'éviter de reproduire les maux est-allemands ${ }^{11}$. Dans ce contexte, les cheminots ont été réceptifs à la critique de la gestion monopolistique du rail. Le discours gouvernemental s'est appuyé avec profit sur la situation de l'Est et sur les rivalités entre les deux modes d'organisation

10- Heinz Dürr, « Une ère nouvelle pour les chemins de fer allemands : leur restructuration, la chance de l'avenir», Rail international, Bruxelles, 1993, p. 2-7.

11- La Reichsbahn était une des plus importantes entreprises d’État en RDA, avec plus de 200000 cheminots, soit davantage qu'à l'Ouest pour un réseau plus petit. 
ferroviaire. Les syndicats ont été sensibles à des promesses de bénéfices sur le long terme et à la présentation de l'immobilisme comme le pire des scénarios. Le président de la DB a annoncé une augmentation du trafic ferroviaire de $50 \%$ au cours des dix années à venir. Une représentante du GdED confirme le caractère exceptionnel de la réforme : "On a beaucoup vanté un modèle allemand de réforme ferroviaire. Pourtant, celui-ci n'est pas nécessairement transposable dans les autres pays européens. À l'Ouest, nous avons accepté de nombreuses concessions sur le plan social car ce qui comptait c'était avant tout de remettre les chemins de fer de l'ex-RDA à niveau ${ }^{12}$. »

Le $1^{\text {er }}$ janvier 1994, la DB et la Deutsche Reichsbahn (DR) fusionnaient pour créer une société par actions, la DB AG (Deutsche Bahn Aktiengesellschaft). La compagnie devenait une entreprise à statut privé, dont le capital restait détenu par l’État. Elle était progressivement divisée en quatre branches destinées à devenir des sociétés anonymes en 1998, avec, pour chacune, des centres de profit afin d'accroitre l'efficacité interne. Un holding coordonnait l'ensemble. On prévoyait sa privatisation, début 2000, par l'ouverture progressive aux capitaux privés. À partir

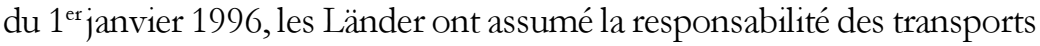
régionaux et locaux, ouverts à la concurrence du secteur privé. La réforme allemande allait plus loin que ne l'imposait la directive 91/440. La DB a salué une réforme qu'elle a qualifiée d'« historique» et de "mesure nécessaire et urgente pour renforcer l'économie nationale $»^{13}$.

Les syndicats allemands ont dû accepter de nombreuses concessions. Ils ont ratifié le processus de privatisation de la DB, ce qui constituait une véritable révolution dans la gestion du réseau ferré. Ils ont donné leur accord à des changements significatifs dans le statut des cheminots. La réforme a introduit l'avancement individuel selon les performances réalisées et une augmentation de la flexibilité dans la gestion du personnel. Les syndicats ont également entériné une réduction importante des effectifs. La DB comptait 355000 cheminots en 1994, il en restait 308000 en 1996. Ces départs ont été négociés sur le long terme jusqu'en 1998 - et la plupart ont été obtenus grâce à des départs volontaires à la retraite et le non-renouvellement des postes. Les licenciements « secs » étaient exclus jusqu'en 1996. Le passage au statut de droit privé était compensé par des rémunérations plus attractives pour les salariés.

12- Entretien réalisé par l'auteur à Paris, en 2003.

13- Deutsche Bahn, The Railway Reform, Frankfurt, DBAG, 1994. 
En échange de leur soutien à la réforme, les syndicats ont obtenu qu'elle n'ait pas de conséquence sur les niveaux de salaires, sur la protection sociale ni sur les retraites. Les syndicats se sont appuyés sur les craintes des Länder, relatives au désengagement financier de l'État fédéral, pour obtenir des garanties concernant la qualité du service public. Les contreparties financières, sociales et juridiques ont permis d'atténuer la portée de la réforme initiale et d'amortir les conséquences sociales de la privatisation. Mais la réforme entrée en vigueur le $1^{\text {er }}$ janvier 1994 n'était pas achevée : elle devait se poursuivre par la négociation entre la compagnie et les syndicats. Ainsi, en mars 1996, une nouvelle convention collective a été signée : elle prévoyait un assouplissement dans le décompte du temps de travail en échange de la préservation de 11000 emplois menacés. La réduction des emplois continuait: la direction visait à atteindre un effectif de 220000 cheminots en 1997 et 150000 à l'horizon de l'an 2000, soit la suppression de plus de la moitié des postes de travail.

\section{Une « menace pour les services publics »}

Contrairement aux syndicats allemands, les syndicats français n'ont pas donné leur accord aux réformes européennes ni négocié leur application. Ils ont exprimé leur opposition à la libéralisation du rail. Le rejet du projet européen et la dénonciation de ses conséquences sociales et économiques ne se sont jamais démentis depuis 1991. Les six syndicats de cheminots ont unanimement désapprouvé la directive 440. Une unité s'est dessinée sur cette question, unanimité suffisamment rare pour être soulignée.

Les discours européens mettant l'accent sur la nécessité de «sauver le rail » furent perçus comme illégitimes. Les syndicats français estimaient que, pendant de nombreuses années, eux seuls s'étaient opposés à la fermeture de lignes, tandis que l'État et la SNCF se contentaient de gérer un déclin annoncé ${ }^{14}$. Deux conceptions différentes de la survie du rail s'affrontaient : pour les partisans de la déréglementation, sa renaissance passait par l'amélioration de sa productivité et de sa rentabilité commerciale et financière, tandis que, pour les syndicats français, sauver le rail signifiait accroitre le volume et la qualité du service par une politique de modernisation des infrastructures et d'investissements ${ }^{15}$. Les syndicats ont insisté sur le sous-investissement chronique dans le

14- En 1991, la SNCF prévoyait de supprimer 4800 emplois pour 1992, après une réduction de 2800 postes en 1991.

15- En 2003, la SNCF a commandé 400 locomotives diesel : cela faisait trente ans qu'elle n'avait pas remplacé ses locomotives fret. 
fret - secteur négligé par la SNCF depuis les années 1960. Le parc fret de la SNCF est vétuste : les locomotives électriques ont en moyenne 27 ans d'âge et les diesels 34. Les syndicats ont également souligné que le débat sur la productivité du rail était faussé tant que le transport routier de marchandises était avantagé et ne payait pas l'intégralité de ses coûts (sociaux, environnementaux ou en matière d'utilisation des infrastructures ${ }^{16}$. La fin de cette concurrence déloyale était considérée comme un préalable indispensable à toute réforme du fret ferroviaire.

Les syndicats ont rejeté le projet européen dans son ensemble car ils estimaient qu'il menaçait une valeur cardinale de l'organisation du rail, le service public. Cette notion implique une égalité d'accès au service pour tous les citoyens, l'universalité et la continuité du service et l'obligation d'exploiter les lignes non rentables. La directive 91/440, en permettant la libre concurrence et l'accès du réseau à de nouveaux opérateurs, entrait en contradiction directe avec cette notion. Les syndicats ont dénoncé les possibilités d'éviction de la compagnie nationale et la recherche d'une rentabilité financière de court terme pour les nouveaux opérateurs.

La distinction comptable entre les activités rentables et celles relevant du service public a été analysée comme une menace pour l'unité de la SNCF. La volonté de conserver un réseau intégré garantissait, aux yeux des syndicats, la pérennité de la péréquation entre les lignes et les activités rentables (les branches voyageurs) et celles qui sont déficitaires (le fret). Les syndicats s'étaient opposés, lors de la décennie précédente, à une politique qui aurait privilégié le TGV au détriment des lignes classiques, dénonçant la mise en place d'une France ferroviaire « à deux vitesses ». La directive venait ainsi bouleverser des valeurs et des compromis sociaux historiques. Georges Ribeill a montré comment les cheminots français lient service public, entreprise publique et garanties statutaires : modifier un élément revient à rompre cet équilibre social ${ }^{17}$. La séparation des activités conduirait à la multiplication des statuts de cheminots, produisant ainsi de nombreux perdants (les salariés travaillant dans le fret, les ouvriers de la maintenance et de l'entretien, déjà soustraités).

La notion de service public est cardinale dans les représentations des cheminots français. Elle est au cœur d'un «contrat républicain» codifié à la Libération. Il relève du mythe identitaire et est revêtu à ce

16- Le budget de la SNCF englobe le financement de ses infrastructures.

17- « Du malaise social à la cassure corporative », Travail, $\mathrm{n}^{\circ} 31$ (1994), p. 37-62. 
titre d'une «dimension sacrée $»^{18}$. L'attachement au service public est également à relier à l'interventionnisme étatique, traditionnel dans les transports. Selon les syndicats, la réussite du TGV est indissociable d'un engagement étatique fort et d'une véritable politique industrielle. De nombreux militants syndicaux ont souligné que « l'invention du TGV ou d'Airbus, dans le contexte de concurrence souhaité par Bruxelles, serait impossible $»^{19}$. La directive européenne était qualifiée par la CGT «d'arme redoutable contre le service public et contre les cheminots $»^{20}$. Contrairement aux syndicats allemands, les organisations françaises ont vu dans l'application de la libéralisation européenne un processus qui contribuait à renforcer des tendances déjà décriées au niveau national. La réforme conduirait à « saucissonner la SNCF $»^{21}$ et à « démanteler la compagnie historique $»^{22}$.

Les réticences syndicales ont été renforcées par l'absence de débat national sur le contenu des textes communautaires. Les différents gouvernements de 1991 à 1999 ont adopté un double discours sur les réformes européennes, discours qui consistait à les approuver à Bruxelles, tout en les stigmatisant à Paris ${ }^{23}$. Les gouvernements français n'ont pas échappé à l'affirmation des thèses libérales dans la gestion des transports pendant les années 1980. Mais, bien que relativement favorables aux projets européens, ils se trouvaient dans l'obligation de composer avec l'attachement de la société française ${ }^{24}$ et des cheminots au service public. Les gouvernements français ont critiqué les propositions européennes tout en faisant adopter progressivement certaines réformes.

18- Danielle Tartakowsky, Le Pouvoir est dans la rue : crises politiques et manifestations en France, Paris, Aubier, 1998, p. 221.

19- Entretiens à la CFDT-Cheminots, Paris, mars 2000.

20- La Tribune des cheminots, le 10 juillet 1992, p.10.

21- Entretiens avec des cheminots français à Paris manifestant contre les projets ferroviaires européens, le 19 octobre 2000.

22- Sophie Béroud, Cégolène Frisque, Trente entretiens auprès d'adhérents de FO en 1995 , Paris, FNSP-Cevipof, 1995.

23- Anne-Cécile Douillet, Dirk Lehmkuhl, « Strengthening the opposition and pushing change: the paradoxical impact of Europe on the reform of French Transport», Differential Europe, op. cit., p. 99-131.

24- $75 \%$ des personnes interrogées pensent que l'intervention de l'État dans les transports favorise un développement plus harmonieux du territoire, $50 \%$ considèrent que sans l'État les services ne seraient pas mieux assurés et les tarifs augmenteraient, $L e$ Monde, 13 avril 1995. 
Le développement d'une rhétorique anti-bruxelloise et l'opposition à «l'Europe libérale » permettait de rendre les réformes nationales plus acceptables $^{25}$. Dans les pays où les relations professionnelles sont traditionnellement conflictuelles, cette stratégie gouvernementale n'est pas rare. Les gouvernements sont enclins à utiliser la contrainte européenne pour faire adopter des mesures impopulaires ${ }^{26}$. Par conséquent, à la différence des autres pays du nord de l'Europe, la réforme communautaire sera imposée aux cheminots non pas au nom des résultats économiques qu'elle est supposée engendrer mais uniquement comme une contrainte extérieure à laquelle le gouvernement s'efforce de résister.

Conscients des résistances syndicales, les gouvernements français ont pesé sur le processus décisionnel européen pour freiner l'application de la directive 91/440. Elle n'entre en vigueur qu'en 1995, soit avec deux ans de retard. Le gouvernement de $M$. Balladur a - discrètement - signé un décret, à la fin de son mandat, entre les deux tours de l'élection présidentielle ${ }^{27}$. La réforme de 1995 a donc été relativement modérée ${ }^{28}$. Il a fallu attendre 1997 pour que soit créé le gestionnaire des infrastructures ferroviaires, Réseau ferré de France (RFF), alors que celui-ci était institué en 1994 en Allemagne. L'application de la directive a été minimale : la séparation entre les branches d'activités n'était que comptable. Le monopole de la SNCF a été préservé dans les faits. Mais la création de RFF en 1997 établissait définitivement le cadre législatif nécessaire à l'entrée de concurrents à la SNCF. L'arrivée d'une coalition de gauche au pouvoir en 1997 a entretenu l'espoir chez les cheminots d'une « réforme de la réforme », promise par le ministre communiste des Transports, M. Gayssot. Les syndicats réclamaient l'abolition de RFF. Jouant sur les mots, le ministre a promis que «lui en place, aucun autre train ne remettra en cause le monopole de la SNCF ».

Présentée ainsi, cette réforme n’a été justifiée par aucune perspective positive nouvelle pour les salariés. La théorie des organisations nous enseigne que les groupes sociaux ne sont pas opposés par nature au changement. Mais, contrairement à leurs homologues allemands, les gouvernements français n'ont pas mis l'accent sur la renaissance du trafic à l'échelle européenne. La réforme n'a pas été introduite comme un

26- Guillaume Devin, Guillaume Courty, La Construction européenne, La Découverte, 2001, p. 16 ; Christian Lequesne, Paris-Bruxelles : Comment se fait la politique européenne de la France, Presses de la FNSP, 1993, p. 54.

27- Décret d'application de la directive 91/440/CE, Journal officiel du 10 mai 1995.

28- Jean-Charles Loupien, «L'adaptation des chemins de fer français aux exigences du marché unique », Cabiers juridiques de l'électricité et du gaz, janvier 1996, p. 1-6. 
défi positif mais comme une obligation imposée de l'extérieur. La déréglementation n'était appuyée par aucune stratégie de reconquête. Aucun projet mobilisateur ne venait étayer les arguments des partisans de la réforme.

Dans ce contexte, la dénonciation des travers de la SNCF a été perçue comme une attaque en règle contre les cheminots ${ }^{29}$. Malgré le déclin marqué du rail, la France était dans une situation relativement favorable, comparée à ses partenaires européens. Le fret y conservait une part de marché supérieure de $70 \%$ à celle de la moyenne communautaire $(26,5 \%$ contre $15,4 \%)$ et les comparaisons internationales en unités kilométriques par agent étaient favorables à la France ${ }^{30}$. C'est dans le pays où la déréglementation posait le moins de problèmes de productivité qu'elle a soulevé les réticences syndicales les plus grandes.

Contrairement aux débats en Allemagne où la directive européenne a agi comme un stimulant supplémentaire à la réforme nationale, en France, elle fut analysée comme une volonté propre des organes communautaires. Ce double discours gouvernemental, cette posture anti-bruxelloise, ces décrets adoptés en catimini illustrent les mauvaises relations professionnelles en France et le rapport complexe entretenu avec les institutions communautaires. La manière dont la réforme a été conduite s'apparente à un jeu d'où personne ne sort gagnant : aucun syndicat n'a donné son accord, l'application de la directive n'a pu être négociée en échange de contreparties sociales, juridiques ou financières. La réduction des effectifs a entretenu la dégradation du climat social. La libéralisation du rail est passée en force et avec des délais plus longs, au prix de nombreuses grèves et d'une stigmatisation des politiques communautaires par les gouvernements nationaux. De 1991 à 1996, le double discours gouvernemental a conforté les syndicats dans l'idée d'un statu quo. Il a contribué à radicaliser leur position de départ. Leur réticence au changement fut d'autant plus forte que la transformation de la SNCF se faisait sans leur assentiment. La méfiance s'est installée, les syndicats soupçonnant le gouvernement d'appliquer la réforme sans le dire. En Allemagne, les termes du débat étaient clairs et sans surprise car un cap avait été fixé. Du côté des syndicats français, une grande incertitude dominait et les rejetait dans un rôle d'opposition à tout changement. Les mauvaises relations professionnelles en France ont joué un

29- Henri Cuq, Dominique Bussereau, Une nouvelle donne pour la SNCF [rapport de commission d'enquête parlementaire], Paris, Assemblée nationale, 2 vol., coll. « Les documents d'information de l'Assemblée nationale », 1994, vol. 1 (rapport), p. 97-103.

30- Christian Stoffaës, Services publics comparés en Europe : exception française, exigence européenne, Paris, La Documentation française, 1995, p. 103 et 109. 
rôle déterminant, si l'on considère, comme Bruno Jobert, que « la réception de nouveaux diagnostics et de nouvelles solutions dépend en fin de compte de la capacité [du gouvernement] à fixer les termes d'un nouveau compromis entre les différents acteurs composant les réseaux de politiques publiques $»^{31}$.

Les réticences des compagnies ont alimenté l'opposition syndicale à la libéralisation. Dans les pays d'Europe du Sud, les compagnies ont exprimé leur opposition à la déréglementation. Elles ont mis en avant une stratégie de coopération entre les réseaux. Le Thalys et Eurostar ont été cités comme des exemples de coopération réussie. Les syndicats opposés à la réforme ont vu leurs analyses confortées par le discours de leur compagnie. Le président de la SNCF qualifiait la concurrence européenne de "grave erreur» reposant sur des "postulats d'ordre idéologique $»^{32}$, tandis que le président de la DB plaidait pour l'ouverture des réseaux. Cependant, à ce stade, il est important de souligner un malentendu entre les syndicats et la direction de la SNCF. Celle-ci rejetait la libéralisation afin de gagner du temps. La mise en concurrence était perçue comme inévitable, une fois acceptée par d'autres pays européens.

\section{Le coût social des réformes}

La réorganisation du rail en Europe a eu de lourdes conséquences sociales. Elle s'est traduite par d'importantes pertes d'emplois. En France, les effectifs sont passés de 194000 en 1992 à 177900 en 1995. En Allemagne, c'est plus de la moitié des postes qui a disparu entre 1990 et $1998^{33}$ (tableau 1).

31- Bruno Jobert (dir.), Le Tournant néo-libéral en Europe. Idées et recettes dans les pratiques gouvernementales, Paris, L'Harmattan, 1994, p. 19.

32- Lettre du 20 mars 1991 au commissaire chargé des Transports, La Vie du rail, 18 avril 1991 ; Jacques Fournier, Le Train, l'Europe et le service public, Paris, Odile Jacob, 1992.

33- Roberto Pedersini (Fondation régionale Pietro Seveso), et Marco Trentini (Ires Lombardia), Comparative study on industrial relations in the rail transport sector, Observatoire européen des relations industrielles (OERI/EIRO), 28 mars 2000, en ligne :

http://www.eurofound.europa.eu/eiro/2000/03/study/tn0003277s.html\#contentpage 
Tableau 1. Évolution du nombre d'emplois dans les chemins de fer, 1990-1998

\begin{tabular}{|l|c|c|c|}
\hline \multicolumn{1}{|c|}{ Pays } & \multicolumn{2}{c|}{ Nombre d'emplois, en milliers } & \\
\hline & 1990 & 1998 & \\
\hline Autriche & 66,9 & 53,5 & \\
\hline Belgique & 45,2 & 40 & \\
\hline Danemark & 20,4 & 10,9 & \\
\hline Finlande & 20,2 & 13,8 & \\
\hline France & 202,1 & 175 & \\
\hline Allemagne & 482,3 & 209,6 & \\
\hline Grèce & 13,5 & 10,9 & \\
\hline Irlande & 11,8 & 10,7 & \\
\hline Italie & 200,4 & 118,3 & \\
\hline Luxembourg & 3,5 & 3,1 & \\
\hline Pays-Bas & 26,2 & 25,8 & \\
\hline Portugal & 22,1 & 10,6 & \\
\hline Espagne & 49,7 & 35 & \\
\hline Suède & 20,8 & 11,1 & \\
\hline Royaume-Uni & 154,7 & - & \\
\hline
\end{tabular}

Source : Roberto Pedersini (Fondation régionale Pietro Seveso), et Marco Trentini (Ires Lombardia), Comparative study on industrial relations in the rail transport sector, Observatoire européen des relations industrielles (OERI/EIRO), 28 mars 2000.

La réforme a également eu des répercussions importantes sur le statut des salariés. Le personnel embauché était désormais sous contrat de droit privé et n'était plus fonctionnaire (en Allemagne). L'unification des anciens statuts des cheminots en Allemagne a débouché, dans la plupart des domaines, sur une harmonisation "par le bas », notamment en matière de garanties d'emploi ${ }^{34}$. L'adoption des méthodes d'organisation et de gestion du secteur privé a accru la précarisation des emplois (recours plus fréquent au travail à temps partiel, par exemple).

34- Serge Schaeffer, "Les garanties individuelles des cheminots et les mutations du secteur ferroviaire au sein de l'Union européenne », 1997, thèse, université de Strasbourg III, p. 143-144 ; Gérard Röder, Jean-Gabriel Recq, « Restructurations et réductions de personnel en Allemagne », Droit social, mai 1994, n 5, p. 502-506, p. 502. 
On peut avancer plusieurs motifs d'explication de ces divergences face aux réformes européennes. Outre la qualité des relations professionnelles, on compte les niveaux de satisfaction de la population à l'égard des compagnies et le degré d'attachement au service public. Si tous les syndicats se réfèrent à la notion de service public, certaines organisations se montrent favorables à des missions de service public gérées par des entreprises privées (sur le modèle suédois) alors que cette idée suscite l'hostilité des syndicats français. En Allemagne et dans les pays scandinaves, il n'y a pas d'attachement aussi fort à la gestion publique. D’autres systèmes de péréquation existent comme celle des ressources fiscales entre Länder allemands. Le dialogue social se déroulant principalement dans les entreprises, on ne constate pas d'exemplarité sociale du service public. En outre, le fonctionnaire allemand est « moins lié à une idée républicaine qu’à une idée impériale qui n’est guère populaire $»^{35}$. En Italie, le service public pâtit d'une mauvaise image pour des raisons politiques (clientélisme, corruption, népotisme, etc.) ${ }^{36}$.

Une autre différence a structuré les réactions syndicales : le rôle de l'État dans l'économie des transports depuis les années 1970. Les syndicats allemands ont exprimé leurs craintes d'un désengagement de l'État. Mais le contexte particulier de la Réunification et le soutien des Länder ont permis aux syndicats d'obtenir des contreparties financières et des garanties juridiques fortes. Dans les autres pays européens attachés au rôle de l'État, l'absence de concertation syndicale sur ce sujet a rendu les syndicats encore plus méfiants vis-à-vis de la libéralisation. Dans les États faiblement interventionnistes dans les transports, la directive a moins été perçue comme un bouleversement des rapports de force et traditions politiques antérieures. La remise en cause du rôle de l'État ou les débats sur le service public n'ont pas eu la même intensité que dans les pays du sud de l'Europe.

Des contextes géographiques (pays de transit ou périphérique, densité urbaine) ou économiques différents ont été autant d'éléments qui ont pesé sur la stratégie des syndicats. Les réformes nationales ont donc été différentes selon l'ampleur de la séparation, le calendrier d'adoption de la directive $91 / 440$, etc. Leurs conséquences sont disparates : la réforme en Allemagne a entraîné l'unification des statuts de cheminots ; au Royaume-Uni, elle a favorisé leur multiplication. Serge Schaeffer

35- Yannick Moreau, Bruno Macquart, Entreprises de service public européennes et relations sociales : l'acteur oublié, Paris, ASPE Europe éditions, 1996, coll. " prospective », p. 156.

36- Michel Le Duc, L'Europe en bref: transports et voies de communication, Arles, Actes Sud, 1997, p. 53. 
note que c'est « la première fois, dans ce secteur très structuré que les évolutions divergent à ce point, tant au niveau des structures internes que des conditions d'emploi du personnel $»^{37}$. Dans l'application d'une directive européenne, les traditions et les débats nationaux restent déterminants.

\section{La cacophonie syndicale en Europe}

Sur des enjeux sociaux similaires (emplois, statut, protection sociale), les syndicats ont adopté des points de vue éloignés. Pourtant, ce constat ne suffit pas à expliquer l'absence de rapprochement. Il existait initialement des réticences syndicales générales à la libéralisation qui auraient pu constituer la base d'un dénominateur syndical commun : tous les syndicats européens ont souligné les risques de fragmentation du réseau, de fermeture des lignes déficitaires, de négligence en matière de sécurité, le sous-investissement dans le secteur, etc. L'histoire de l'intégration européenne n'est jamais celle d'une convergence a priori des intérêts nationaux. Une volonté commune peut triompher d'intérêts divergents. La construction communautaire a abouti à l'atténuation des antagonismes nationaux et à la négociation de compromis acceptables par tous. Ce qui a été possible entre des États rivaux de longue date peut l'être de syndicats qu'opposent des stratégies politiques différentes.

Or, au début de la décennie 1990, ces compromis syndicaux minimaux paraissaient impossibles. Aux différences nationales se sont ajoutés des phénomènes de stigmatisation réciproque. Les organisations défendant une stratégie différente ont été perçues comme des adversaires. Cette disqualification de l'autre a contribué à rendre le dialogue impossible entre deux camps : ceux qui acceptaient de négocier la déréglementation et ceux qui tentaient d'y résister. L'absence de discours syndical européen est liée au refus obstiné de reconnaissance par les uns de la légitimité des options retenues par les autres.

Le contraste entre les deux modèles de réaction syndicale fut régulièrement utilisé par les gouvernements, la presse et, parfois, les compagnies, pour stigmatiser les refus des syndicats nationaux. En France, la presse a fréquemment commenté «la prouesse allemande $»^{38}$. Elle soulignait que la restructuration du rail, avec la réduction du nombre de cheminots de 400000 à 200000 agents, s'était accomplie «sans aucune grève ». Le "modèle allemand » était abondamment cité en

37- Serge Schaeffer, thèse citée, p. 78

38- «Le retour du modèle allemand », Le Monde, 19 septembre 1995. 
exemple $^{39}$. Les syndicats français ont nourri un grand ressentiment à l'égard des syndicats allemands. Ils furent perçus à la fois comme un affaiblissement des stratégies et luttes françaises, comme une «trahison » de la cause syndicale européenne et à une compromission avec des logiques libérales décriées. Ils leur contestaient le caractère d'organisation défendant les intérêts des salariés. Un représentant de la CGT-cheminots témoigne : "Je me souviens du nombre de réunions au siège de la FST ${ }^{40}$ où, après des heures de discussions, on n'aboutissait à rien. Bien souvent, on s'est dit que l'on se sentait plus proche et qu'on avait plus d'affinités avec des dirigeants de la SNCF qu'avec des syndicats scandinaves : c'est un comble ${ }^{41} ! »$

De leur côté, les syndicats allemands considéraient les stratégies des syndicats français comme « irresponsables ». Ils ont perçu leur discours comme un obstacle à l'obtention de contreparties sociales européennes par la négociation. Ils n'hésitaient pas à condamner leur stratégie ${ }^{42}$, dénonçant un comportement égoïste et de "passager clandestin ». Le refus d'ouverture des réseaux était assimilé à du protectionnisme alors que, dans le même temps, la SNCF entreprenait de conquérir des lignes sur les réseaux nordiques et allemands. Les logiques de stigmatisation réciproque et de concurrence entre les positions syndicales nationales ont fait de chacun un obstacle à la stratégie de l'autre.

39- Philippe Ricard, "La relance du chemin de fer en Allemagne dépasse les objectifs ", Le Monde, 27 avril 1995 ; «L'exemple allemand », La Vie du rail, $\mathrm{n}^{\circ} 2515$ (oct. 1995), p. 10-20 ; Maurice Kopecky, « Deutsche Bahn AG et le défi de l'an 2010 ou les mutations du paysage ferroviaire allemand », Transports, 1994, p. 314.

40- Fédération syndicale européenne des salariés du transport, membre de la Confédération européenne des syndicats (CES).

41- Entretien à Paris, en 2002

42- GdED [Gewerkschaft der Eisenbahner Deutschlands], Die Bahnreform : Herausforderung für die Eisenbahner, 1996. 